\title{
Natural technologies for sustainability: accelerating implementation
}

erformed by Epta in its laboratories in Limana (BL) in Italy.

The Life-C4R technologies are

- FTE2.0 (Full Transcritical Efficiency) A device that allows ellimination of the superheat, thus increasing the evaporation temperature in the MT cabinets evaporators (fresh food products), obtaining a considerable reduction in the energy consumption of the cooling power rack.

- ETE (Extreme Temperature Efficiency)

A sub-cooler using natural refrigerant $\mathrm{CO}_{2}$ integrated into the refrigeration system, extending the range of use of the $\mathrm{CO}_{2}$ system in the hot climates and with temperatures above $40^{\circ} \mathrm{C}$

The Life-C4R technologies have been implemented in seven food retail stores in Italy, Romania and Spain.

These pilot installations were important in proving the reliability, performance and energy consumption of the C4R system in different conditions:

- Store surface, from 1000 to 6000 sqm - Climatic conditions, from mild to extremely hot temperatures

- Different countries and with different habits, knowledge, skills, etc.

\section{Data and results}

The pilot projects were observed over more than a full year and aimed to verify the reliability and measure performance operation and consumption in all seasons. The pilots clearly show that the Life-C4R technologies guarantee a reduction in energy consumption ranging from 15 per cent to 23 per cent on an annual basis-much higher than initially expected.

The key outcome of the pilot store trials is that the Life-C $4 \mathrm{R}$ technologies using $\mathrm{CO}_{2}$ natural refrigerant always guarantee very efficient operation regardless of the outside temperature. The data acquired from the seven pilot stores allowed to build a model to predict the behaviour of the FTE2.0

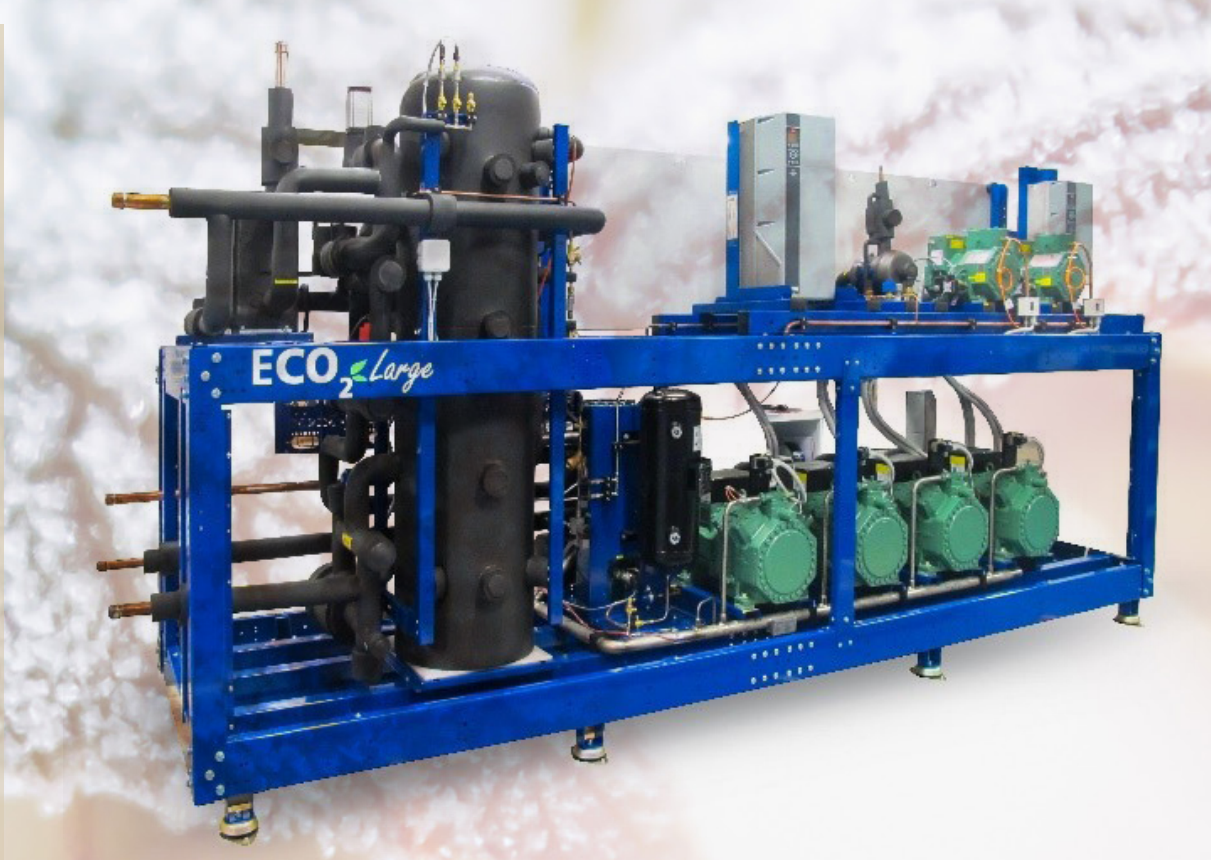

Figure 1: Transcritical $\mathrm{CO}_{2}$ power rack for medium-large stores with incorporated FTE2.0 and ETE.

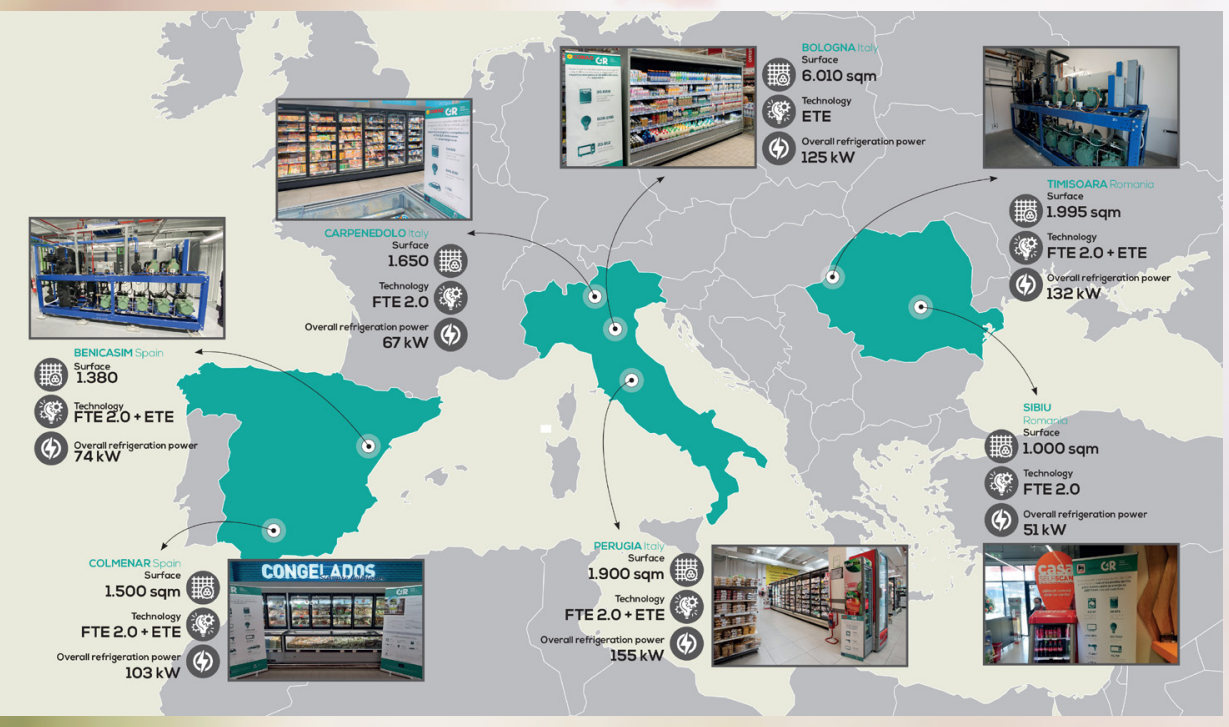

A ETE systems in any other condition. calculation of the overall carbon footprint A computational analysis tool has been of the system during its entire useful life A crom cradle to grave" considering:

The LCCP analysis

To monitor and evaluate the global carbon footprint of these innovative $\mathrm{CO}_{2}$ solutions, Epta performed a full gas emissions of according to the standards of LCCP (Life Cycle Climate Performance). phase of ndustrialdesignanddevelopment on performance tests and fine-tuning
LCCP is a method that allows precise a) direct emissions deriving from the loss of refrigerants into the atmosphere during the operation and disposal of the equipment;

b) indirect emissions of greenhouse gases in relation to the production of electricity that powers the equipment; c) incorporated emissions, related to the processes of production, transport and end-of-life disposal of all materials, the equipment and the refrigerant. 


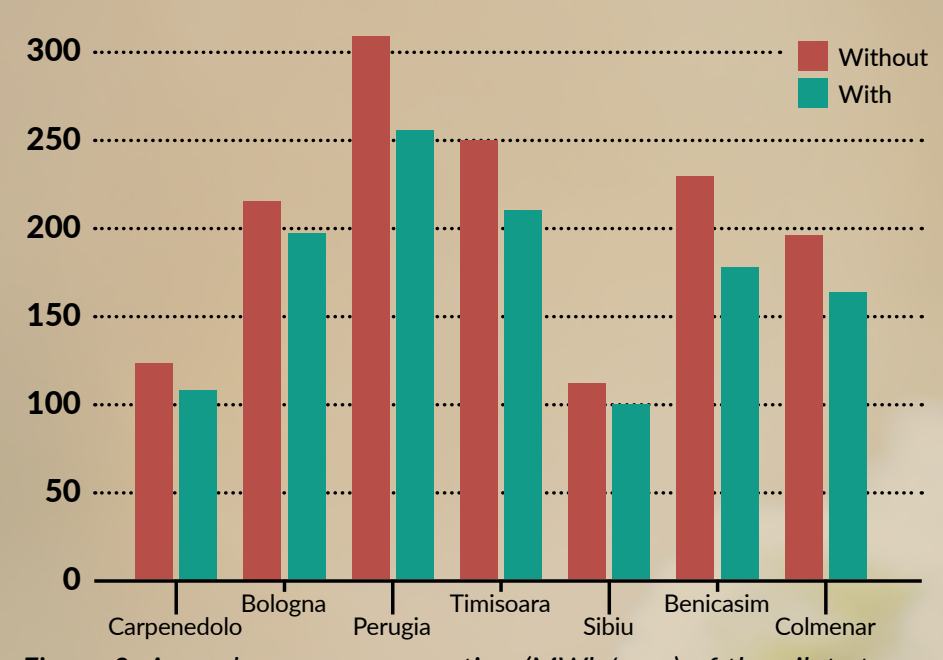

Cigure 3: Annual energy consumption (MWh/year) of the pilot stores

Figure 3: Annual energy consumption (MWh/year) of th
with (green) and without (red) Life-C4R technologies.

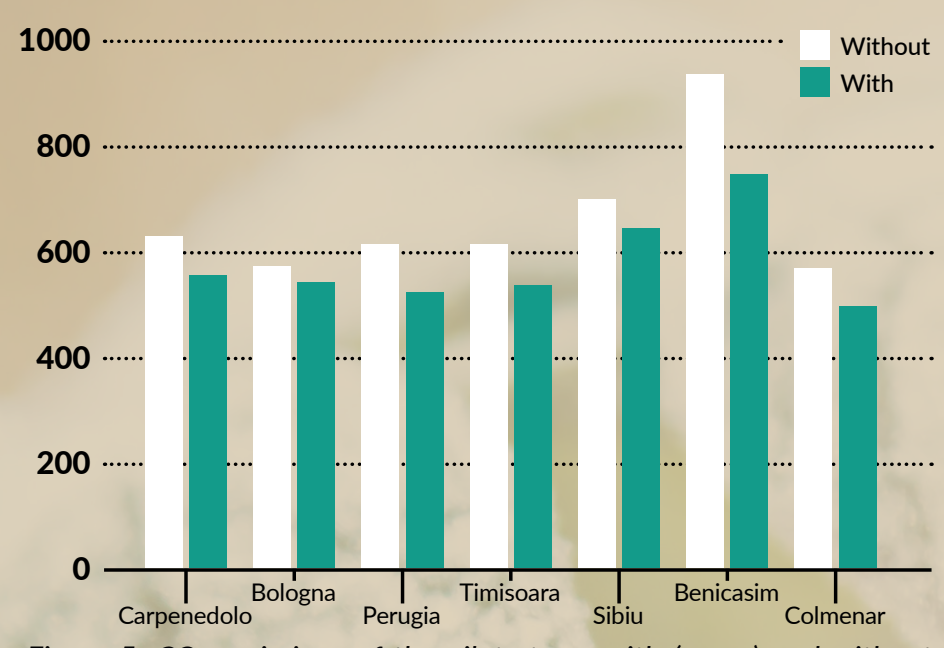

Figure 5: $\mathrm{CO}_{2}$ emissions of the pilot stores with (green) and without Figure 5: $\mathrm{CO}_{2}$ emissions of the pilot stores with (
(white) Life-C4R technologies ( $\left(\mathrm{kg}^{\mathrm{CO}}\right.$ eq per $\mathrm{kWh}$ ).

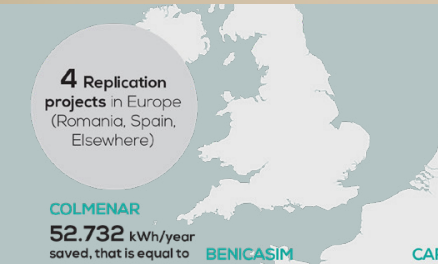

P. PROJECT PILOTS

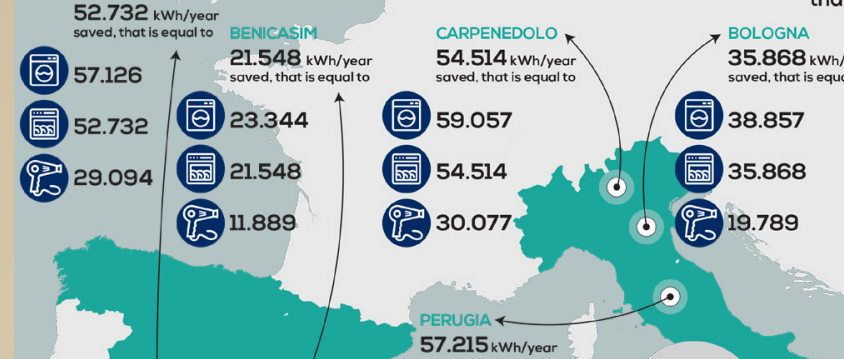
at guarantee remarkable energy savings
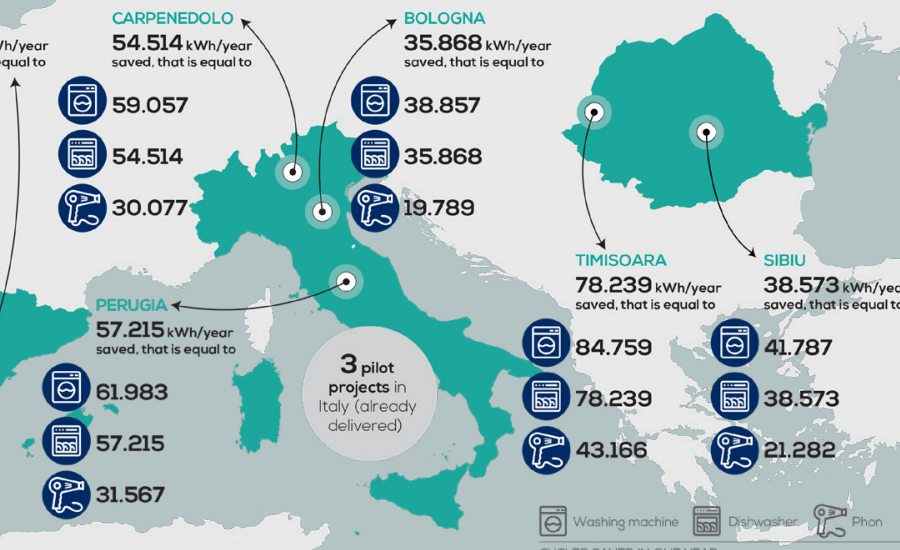

国 wemangative 圆

Figure 4: Energy savings in the Life-C4R pilot stores ( $\mathrm{kWh} / \mathrm{year}$.

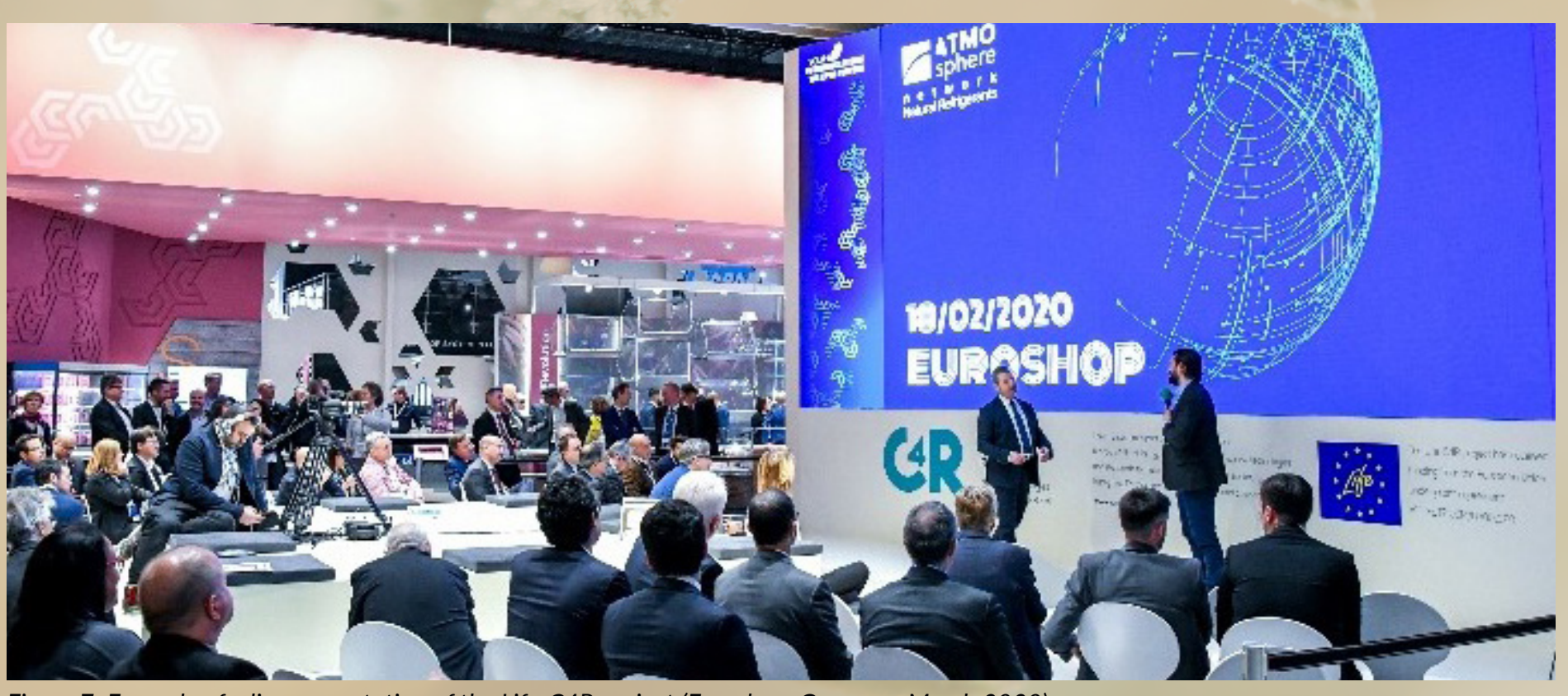

Figure 7: Example of a live presentation of the Life-C4R project (Euroshop, Germany, March 2020.

alysis shows that the use of Lifeglobally by up to 20 per cent.

n particular, it has been observed that the and most of the impacts originate from the energy consumption of the system, which is very positive as most of the electricity will be produced from renewable sources in the future.

\section{Communication and} networking activities

In the spirit of the LIFE programme, an extensive communication activity has Life-C4R technical solutions and their Furthermore, a process of global equipped with modern technologies, technologies after the end of the project.

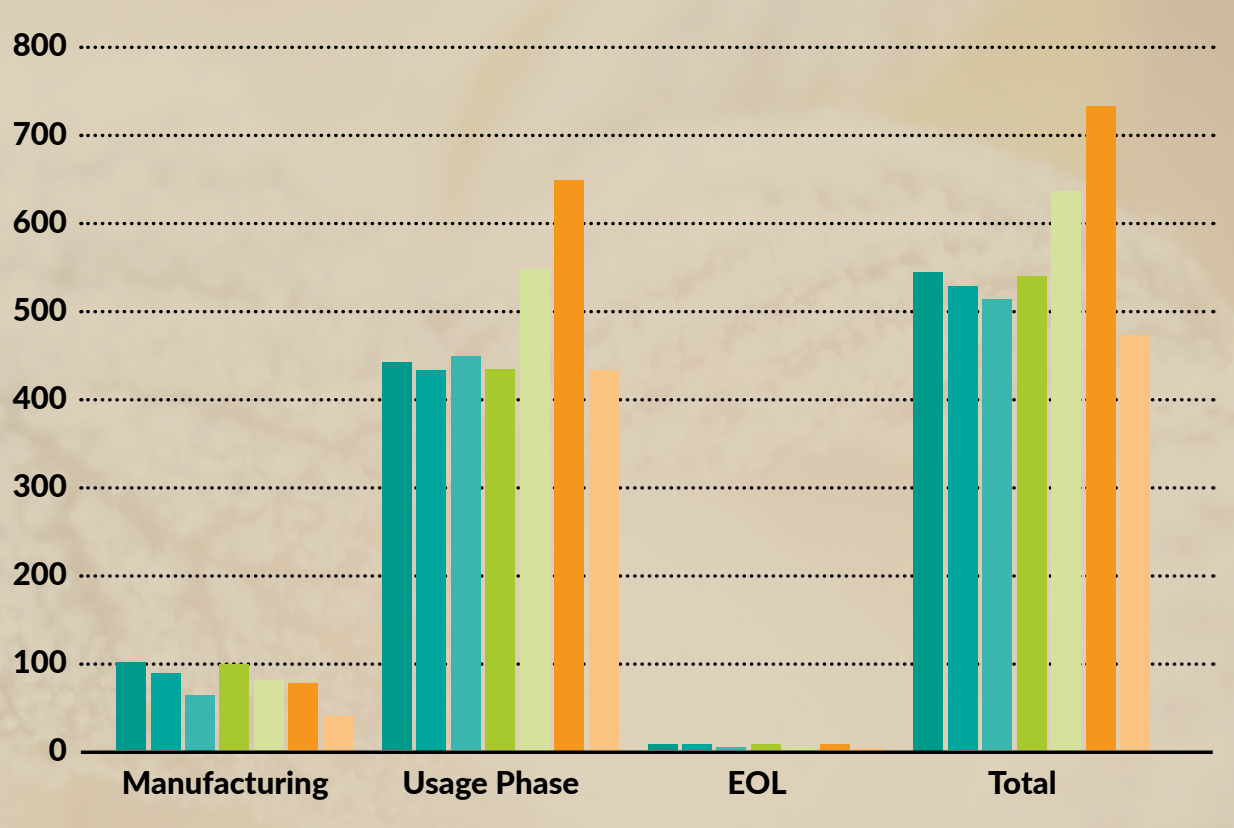

PROJECT NAME

Carbon 4 Retail Refrigeration (C4R)

PROJECT SUMMARY

The Life-C4R project is an international

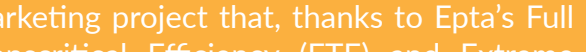
Transcritical Effciency (FTE) and Extreme
Temperature Efficiency (ETE) systems, will substantially contribute to replacing HCFC and HFC greenhouse refrigerants with $\mathrm{CO}_{2}$. inc any external temperature, allowing 10 per
and cent energy and 30 per cent installation and

PROJECT PARTNERS

Epta is a multinational group specialising that operates excellent performances in the Retail, Ho.Re. $\mathrm{Ca}$ and food and beverage sectors. Epta lberia is specialised in the ints and the
missioning of retailers refrigerated sites.

DAAS is an engineering, maintenance and project management company that provides
turnkey projects for commercial retail.

environmental implications to the wider maintenance of existing supermarkets public of professionals and ordinary and hypermarkets that are continuously people, intemational organisations, dispersing a lot of refrigerant into the policymaks and the gencer, policymakers and the general public, conferences, seminars, networking and Highly efficient sustainable technologies professional training meetings, promoting such as Life-C CR offer the possibility and supporting the massive use of natural to initiate a structural programme of refigerants to achieve significant energy savings and to combat climate change. existing store retrofits, to replace the highly climate-impacting commercial refigeration systems with new systems involvement and interest has been initiated worldwide to favour a wide diffusion and rapid adoption of Life-C $C R$ climate neutrality by 2050 .

Replacing an HFC system with natural refrigerant $\mathrm{CO}_{2}$ means reducing the direct greenhouse effect up to 4000 times and obtaining a significant energy upgrade with a consequent reduction in the system's energy consumption.

Additionally, the Life-C4R political implications at the European level involve the F-Gas Regulation (EU 517/2014) review, which will increase its ambitions in response to the climate crisis, defining a long-term sustainable scenario that hopefully will foresee the exclusive use of the lowest GWP refrigerants for the new
equipment and for the maintenance of equipment and for the maintenance of existing installations.

\section{PROJECT LEAD PROFILE}

Epta combines a solid industrial culture, great
competitive and innovative strength and a competitive and innovative strength and a
significant presence worldwide. The group offers the widest and most comprehensive refrigeration, ensuring the supply, installation
reng and maintenance of systems. Epta also has a strong presence within the food and beverage market, working in partnership
with major players in the sector.

Francesco Mastrapasqua

Institutional Affairs Manager
Via degli Alpini, 14, 32020 Limana (BL),

Ii +3904379681

Francesco.Mastrapasquale

eptarefrigeration.com

www.eptarefrigeration.com

in

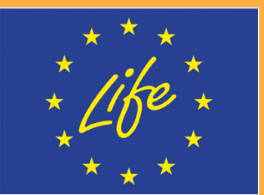

FUNDING The LIFE-C4R project has received funding from
the LIFE programme of the European Union unde-
grant agreement No. LIFE17 CCM/IT/000120.
PROJECT CONTACTS 